\title{
A Structural Learning Algorithm for Neural Networks and Handwritten Zipcode Recognition
}

\author{
Akihiro Takatsuka, Hideki Nio, Kazuhiro Ichikawa and Sueo Sugimoto \\ Department of Electrical and Electronic Engineering, Ritsumeikan University \\ Noji-cho, Kusatsu City, Shiga. 525 Japan
}

\begin{abstract}
The feedforward layered neural networks have been applied to the various classification problem and control. The generalization capability of neural networks is one of the most important properties to be acquired such that it depends on the numbers of units or weights. In this paper, we propose a fast and effective structural learning algorithm to construct a suitable structure for neural networks and apply to the pattern recognition problem for handwritten numerical characters. At every learning step evaluations for unknown data are computed, and decide the stopping time of learning (test set validation). Furthermore we consider the validity to select an optimal neural network model by information criteria.
\end{abstract}

\section{Introduction}

The artificial neural networks have been applied to many problems, because of its parallel processing and learning capability. Especially, the feedforward layered neural network; one of typical network models, have been applied to the pattern recognition problem and control problem, since the back propagation (BP) algorithm had been proposed [1]. However, it is important to acquire the generalization capability: namely, the neural network can present the proper output for unknown data (test data). When the structure of the network is not suited, the generalization capability will be worsen, because neural networks learn input-output patterns as internal representations. The best generalization capability is obtained by trading off the training error and the network complexity (the number of parameters in the network). In order to solve this problem, recently several methods have been proposed to construct a suitable structure, in which the numbers of links (weights) or units are optimized through the learning; an optimal structural method of neural networks by multiplication (addition) and combination of hidden units [2], Optimal Brain Damage (OBD) [3], and the algorithm with forgetting [4]. Also for selecting the optimal network structure model, several methods by using information criteria have been proposed in [5][6][7][8].
In this paper, we propose a fast and effective structural learning algorithm to construct a suitable structure by combining the so-called optimal structural method [2] and OBD [3] (both methods are reviewed in this paper, see appendices A and B, respectively).

And we apply the proposed method to the pattern recognition problem for handwritten numerical characters, specially for the recoginition of the handwritten zipcode. At each learning step in the proposed algorithm, the mean square error (MSE) of the outputs and the successful recognition rate for unknown data are computed, and decide the stopping time of learning by considering these quantities (test set validation). Also simulteneously several information criteria such as AIC (Akaike Information Criterion) and MDL (Minimum Description Length) are computed for training data based upon [5] such that we check the feasibility of applying the method proposed in [5] for selecting optimal neural network model.

\section{A fast and effective structural method}

To construct a suitable structure of a neural network, we propose a fast and effective structural learning algorithm by combining the optimal structural method and OBD. 
When we only apply the optimal structural method, it may be usually that the number of hidden units may become suitable, but unnecessary links (weights) may be remained. Also if hidden units are combined together, at the same instant so many weights; some of them may still play important roles in the network, will be deleted. Therefore when the number of hidden units begins to be decreased in the optimal structural method, we apply OBD to construct a suitable structure of the neural network regarding to the number of links (weights). By such combination of two algorithms, we try to construct a reasonable network architecture for a better generalization capability.

On the other hand, an important problem and question for a pattern recognition problem by applying the $\mathrm{BP}$ algorithm is as follows: when the MSE for training data becomes small in the BP learning process, but no strong correlation between the MSE and the generalization capability (cf. the experimental result of zipcode recognition, see Fig. 1). Therefore when the MSE for the training data begins to be small in the BP learning process, we had better to pay attention to the MSE for unknown data or the successiful recognition rate for unknown data.

From the aspects in the above discussions, the following method is proposed.

1. Until the MSE for training data becomes small, we only apply the optimal structural method assuming that there are some correlation between the error for training data and the generalization capability. The initial number of the hidden units is setted to be two. If the changing rate of the MSE is less than a preassigned threshold, let the number of hidden units be increased twice.

2. In the optimal structural procedure, when the correlation coefficient between two hidden units is large, combine those two hidden units into one unit. Also when the variance of a hidden unit is small, combine the hidden unit into the bias unit.

3. When the number of hidden units begins to decrease, keep this number of hidden units and apply OBD once for getting a better generalization capability.
4. In these learning procedures, the MSE for training data will be usually small. Then at each learning step in the optimal structural procedure, compute the MSE and the successiful recognition rate for "unknown data" so that we look these quantities for evaluating the generalization capability (test set varidation).

5. Decide the stopping time of learning in the optimal structural procedure by considering the MSE and the recognition rate for unknown data.

\section{Zipcode recognition and experimental results}

The proposed method is applied to the recognition problem of handwritten zipcodes which are distributed from Japanese Institute for Posts and Telecommunications Policy [9]. First, before extracting the features from the numerical character image, the image is preprocessed such as removing the noise, transforming the patterns by the cosine function [10], normalizing the size and extracting contour points. Secondly patterns are converted to the feature quantities with directional elements [11]. Lastly numerical characters are classified by three layered neural networks with the proposed method. In this experiment, the three layered neural network model with 100-inputs and 10-outputs units is applied. The extracted features with 100 directional elements are used as input data. The target outputs for $k$-th category under the input for the $n$-th training data are $T_{k}^{(n)}=1$ for $k$-th unit, and $T_{k}^{(n)}=0$ for other units. Five hundred samples of training data and and 1,000 samples of unknown data (test data) are used in this experiment.

\section{Results by Optimal structural method}

Fig.1 and Fig.2 are the experimental results by applying the optimal structural method. Fig.1 shows the relation between the MSE error and the successful recognition rate for unknown data (as an index of the generalization capability) in each learning time. Although both the errors for training data as well as for unknown data are going to be small, the recognition rates for unknown data (test data) are gradually going down. These phenomena tell us that 
we should also pay attention to the MSE and the successful recognition rate for unknown data.

Fig.2 also shows the relation between the change of numbers of hidden units and successful recognition rates for unknown data in each leaning step of the optimal structural method. When the number of hidden units begins to decrease (around 30-th time at the learning step), the recognition rate for unknown data reached the maximum. Although the size of the neural network becomes small in these learning times, the successful recognition rate for unknown data does not go down, where the maximum successful recognition rate for unknown data achieves $94.6(\%)$, and then the number of hidden units are 40. Comparatively, we done the experiment of applying usual the BP learning algoritms where the hidden unit were fixed as constant; 40. Then we obtained the successful recognition rate for unknown data that is $94.3(\%)$.

\section{Results by the proposed method}

When the number of hidden units begins to decrease (from 48 to 47 at around 30 times learning step) in the optimal structural method, fixed up the number of hidden units as 47 and applied the OBD such that we got a neural network which has the better generalization capability. Fig. 3 shows the results of such experiments. After OBD being applied, the usual BP learning algorithm were repeated for under fixing the numbers of units and connections (weights). After OBD applied, the successful recognition rates became a little worse, comparing with applying the optimal structural method only, but after around 20 times learning the recognition rate for unknown data became higher and reached to the maximum value as $95.0(\%)$. In this process we could delete 307 weights from the network which was obtained by the optimal structural method and consisted of 40-hidden units.

Here, we consider the feasibility of applying information criteria in the proposed method. Several methods by information criteria to select optimal number of hidden units have been proposed [5] [6] [7] [8], and they showed some useful results. However it is usually difficult that we can always obtain the maximum likelihood estimates for parameters in network models that are composed of many nonlinear computational elements. Then we consider here the feasibiltiy of applying the information criteria through the experiments of the handwritten numeric character recognition. As one of methods of computing of information criteria: AIC(Akaike Information Criterion) and MDL(Minimum Description Length), we had proposed a simple approach of applying AIC and MDL for choosing the optimal number of hidden units as follows [5].

\section{A simple approach of applying AIC and MDL [5]}

We denote the target value and the output of network as $T_{k}^{(n)}$ and $y_{k}^{(n)}$ on the $k$-th unit of the output layer for the $n$-th training data, respectively. We assume that the error $T_{k}^{(n)}-y_{k}^{(n)}$ is the normal distribution with mean $\mu_{k}$ and variance $\sigma_{k}^{2}$. Then, the $\log$-likelihood function is

$$
l_{k}(\underline{w})=\log \prod_{n=1}^{N} \frac{1}{\sqrt{2 \pi \sigma_{k}^{2}}} \exp \left[-\frac{\left(T_{k}^{(n)}-y_{k}^{(n)}-\mu_{k}\right)^{2}}{2 \sigma_{k}^{2}}\right] .
$$

Since it is almost impossible to obtain the maximum likelihood estimates for the parameters of neural networks, we consider post-training parameters $\underline{\tilde{w}}$ as the approximates of the maximum likelihood estimates $\underline{\hat{w}}$. By using outputs of the post-training neural network $\tilde{y}_{k}^{(n)}$, we have the estimates of mean $\mu_{k}$ and variance $\sigma_{k}^{2}$ as

$$
\begin{gathered}
\hat{\mu}_{k}=\frac{1}{N} \sum_{n=1}^{N}\left(T_{k}^{(n)}-\tilde{y}_{k}^{(n)}\right), \\
\hat{\sigma}_{k}^{2}=\frac{1}{N} \sum_{n=1}^{N}\left(T_{k}^{(n)}-\tilde{y}_{k}^{(n)}-\hat{\mu}_{k}\right)^{2} .
\end{gathered}
$$

If we can assume that each output from units in the output layer is mutually independent and gaussian, the log-likelihood function is given by

$$
\begin{aligned}
l(\underline{\tilde{w}})= & \log \prod_{k=1}^{K} \prod_{n=1}^{N} \frac{1}{\sqrt{2 \pi \hat{\sigma}_{k}^{2}}} \exp \left[-\frac{\left(T_{k}^{(n)}-\tilde{y}_{k}^{(n)}-\hat{\mu}_{k}\right)^{2}}{2 \hat{\sigma}_{k}^{2}}\right] \\
= & \sum_{k=1}^{K}\left\{-\frac{N}{2} \log \left(2 \pi \hat{\sigma}_{k}^{2}\right)\right. \\
& \left.-\frac{1}{2 \hat{\sigma}_{k}^{2}} \sum_{n=1}^{N}\left(T_{k}^{(n)}-\tilde{y}_{k}^{(n)}-\hat{\mu}_{k}\right)^{2}\right\} .
\end{aligned}
$$

Substitute (3) to (4), we have

$$
l(\underline{\tilde{w}})=\sum_{k=1}^{K}\left\{-\frac{N}{2} \log \left(2 \pi \hat{\sigma}_{k}^{2}\right)-\frac{N}{2}\right\} .
$$


Then we have

$$
\begin{gathered}
A I C(P)=-2 l(\underline{\tilde{w}})+2 P, \\
M D L(P)=-l(\underline{\tilde{w}})+\frac{P}{2} \log N .
\end{gathered}
$$

Finally we select the neural network model with the optimal number of hidden units that minimize (6) and (7).

When we apply the above simple approach to the experiment of the zipcode recognition, we compute AIC and MDL at each learning step. We assume the parameters before the number of parameters changed in the optimal structural process as the approximate of the maximum likelihood estimate $\underline{\hat{w}}$. This time we use optimal structural method only. The information criteria are computed by (11) and (12). The successful recognition rate (\%) for unknown 1000 data is shown in Table 1, where "units" represents the number of hidden units, " $E$ " and " $E_{\text {test }}$ " represent the MSE for training data and the MSE for unknown data respectively. In 29 and 46 learning times, the successful recognition rate for unknown data achieve the maximum in the Table 1 , but in these learning times AIC and MDL are not minimum. It is desired when information criteria reach the minimum the generalization capability reachs the maximum. But we cannot strictly to say that information criteria is reasonable for especially pattern recognition problems. Information criteria is composed of two terms: the ordinary training error, plus some measure of the network complexity. The appropriate measure of network complexity is the number of parameters in the network. Therefore if we use optimal structural method, information criteria will be decrease as learning time increase, but the generalization capability will be not always become better.

\section{Conclusions}

We proposed a hybrid method by combining the optimal structural method and OBD. We could effectively delete parameters, and we could architect a reasonable network that has a better generalization capability.

In the case that we can use a large amount of data, it is a simple and reliable method that we compute MSE or recognition rate for unknown data at every learning step and observe those evaluations (test set validation).

In the case that we cannot use a large amount of data, Cross Validation seems to be a useful method. Wada and Kwato[6] proposed an information criterion using Cross Validation, when the maximum likelihood estimation can not be obtained. However it may take huge computation costs. Therefore we should find a simpler method for evaluating the generalization capability of networks. This subject will be investigated in future.

\section{References}

[1] D. E. Rumelhart, et al.: "Parallel Distributed Processing Vol.1:Foundations," MIT Press, Cambridge, MA. (1986).

[2] T. Masuda: "An Optimal Structuring Method of Multi-Layered Neural Networks by Multiplication and Combination of Hidden Units," (in Japanese), Proc. 36th Annual Conference of ISCIE, pp.151$152,(1992)$.

[3] Y. L. Cun, J. S. Denker and S. A. Solla: "Optimal Brain Damage," in D. S. Touretzky(ed.) Advances in Neural Information Processing Systems, Vol.2, pp.598-605, Morgan Kaufman, San Mateo, California (1990).

[4] M. Ishikawa: "A Structural Connectionist Learning Algorithm with Forgetting," (in Japanese), Japanese Society for Artificial Intelligence, Vol.5, No.5, pp.595-603, (1990).

[5] M. Kabe, K. Mima and S. Sugimoto: "Selection of the Number of Units for Neural Networks," Preprints of the 24th ISCIE Symp. on Stochastic Systems Theory and its Applications, pp.85-88, Kyoto (1992).

[6] Y. Wada and M. Kawato: "Estimation of Generalization Capability by Combination of New Information Criterion and Cross Validation," (in Japanese), IEICE Trans., J74-D-II-7, pp.955965(1991).

[7] T. Kurita: "A Method to Determine the Number of Hidden Units of Three Layered Neural Networks by Information Criteria, " (in Japanese), IEICE Trans., J73-D-II-11, pp.1872-1878(1990). 
[8] D. B. Fogel: "An Information Criterion for Optimal Neural Network Selection," IEEE Trans. Neural Networks, Vol.2, No.5, pp.490-497 (1991).

[9] T. Matsui et al.: "State of the Art of Handwritten Mumerical Recognition in Japan, "Proc. the IEEE 2nd Int. Conf. on Document Analysis and Recognition, Tsukuba Science City, Oct. 20-22, pp.391-396 (1993).

[10] J. Guo, et al.: "Recognition of Handwritten Characters Using Pattern Transformation Method with Cosine Function, " (in Japanese), IEICE Trans., J76-D-II-4, pp.835-842(1993).

[11] N. Sun, et al.: "Printed Character Recognition Using Directional Element Feature," (in Japanese), IEICE Trans., J74-D-II-3, pp.330-339(1991).

\section{Appendix A}

\section{Optimal Structural Method[2]}

This is an extended structuring method to improve the generalization capability of a network by uniting the multiplication and combination of hidden units.

At first, set each parameter of the neural network, and initialize the connected weights of layers with small random numbers. The initial number of the hidden units are two.

At each learning step by applying the BP algorithm, compute the output vector $\left\{V_{i p}\right\}$ ( $i$-th hidden unit for the $p$-th learning data) and the mean square error (MSE) and then renew each free parameter. After the second learning, compute the changing rate of MSE; $\Delta E$.

Compute the correlation coefficient $r_{i j}$ of $\left\{V_{i p}\right\}$ and $\left\{V_{j p}\right\}$ :

$$
r_{i j}=\frac{\frac{1}{n} \sum_{p=1}^{n} V_{i p} V_{j p}-\overline{V_{i}} \overline{V_{j}}}{\sqrt{\frac{1}{n} \sum_{p=1}^{n} V_{i p}{ }^{2}-{\overline{V_{i}}}^{2}} \sqrt{\frac{1}{n} \sum_{p=1}^{n} V_{j p}{ }^{2}-{\overline{V_{j}}}^{2}}}
$$

where

$$
\overline{V_{i}}=\frac{1}{n} \sum_{p=1}^{n} V_{i p}, \quad \overline{V_{j}}=\frac{1}{n} \sum_{p=1}^{n} V_{j p},
$$

$n$ is the number of the training data, $V_{i p}, V_{j p}$ are the outputs of $i$-th and $j$-th hidden units for $p$-th data respectively. Compute variance $s_{i}^{2}$ of each output of hidden unit :

$$
s_{i}{ }^{2}=\frac{1}{n} \sum_{p=1}^{n} V_{i p}{ }^{2}-{\overline{V_{i}}}^{2} .
$$

After the second learning, compute the changing rate of the correlation coefficient $\Delta r_{i j}$.

The optimization of the number of hidden units can be carried out as follows.

If all the $\Delta r_{i j}$ are less than the threshold $\lambda_{r}$ and $\left|r_{i j}\right| \geq \theta_{1}$ and $s_{i}{ }^{2}, s_{j}^{2} \geq \theta_{2}$, combine $i$-th and $j$-th hidden unit into one unit. If all the $\Delta r_{i j}$ are less than the threshold $\lambda_{r}$ and $s_{i}{ }^{2}<\theta_{2}$, combine the $i$-th hidden unit with the bias unit. If $\Delta E$ is less than the threshold $\lambda_{E}$ and all the $\Delta r_{i j}$ for $i$ and $j$ are less than the threshold $\lambda_{r}$, let the number of hidden units be twice.

\section{Appendix B}

\section{Optimal Brain Damage (OBD)[3]}

OBD is a method to remove unimportant weights from a network by using second-derivative information.

It would be extremely laborious to compute the strict evaluation of the change of the objective function $E$ caused by deleting a parameter. So we predict the change of the $E(: \delta E)$ by a Taylor series:

$$
\begin{aligned}
\delta E= & \sum_{i} g_{i} \delta u_{i}+\frac{1}{2} \sum_{i} h_{i i} \delta u_{i}^{2} \\
& +\frac{1}{2} \sum_{i \neq j} h_{i j} \delta u_{i} \delta u_{j}+O\left(\|\delta U\|^{3}\right) .
\end{aligned}
$$

where the $\delta u_{i}^{\prime} s$ are the components of the perturbation $\delta U$ of the parameter vector, the $g_{i}^{\prime} s$ are the components of the gradient of $E$ with respect to $U$, the $h_{i j}^{\prime} s$ are the elements of the Hessian matrix $H$ of $E$ with respect to $U$ :

$$
g_{i}=\frac{\partial E}{\partial u_{i}}, \quad h_{i j}=\frac{\partial^{2} E}{\partial u_{i} \partial u_{j}} .
$$

We wish to find a set of parameters whose deletion will cause the least increase of $E$. But it is very difficult to compute directly Eq.(B1), because the matrix $H$ is enormous. Therefore we must use some approximations. Assuming the diagonal approximation for the Hessian matrix, and assuming the external approximation after training has converged, and assuming the quadratic approximation. After all Eq.(B1) is given by 


$$
\delta E=\frac{1}{2} \sum_{i} h_{i i} \delta u_{i}^{2} .
$$

To apply to a neural network, the connection from unit $j$ to unit $i: \omega_{j i}$ is equal to $u_{k}$ for all $(i, j)$. Then saliencies $S_{i j}$ for each weight $\omega_{j i}$ :

$$
S_{j i}=\frac{1}{2} \frac{\partial^{2} E}{\partial \omega_{j i}^{2}} \omega_{j i}^{2} .
$$

Train the network until a reasonable solution is obtained and choose a reasonable network architecture. After that, compute the saliencies, and sort the weights by saliency and delete some low-saliency weights, and again iterate the BP learning.

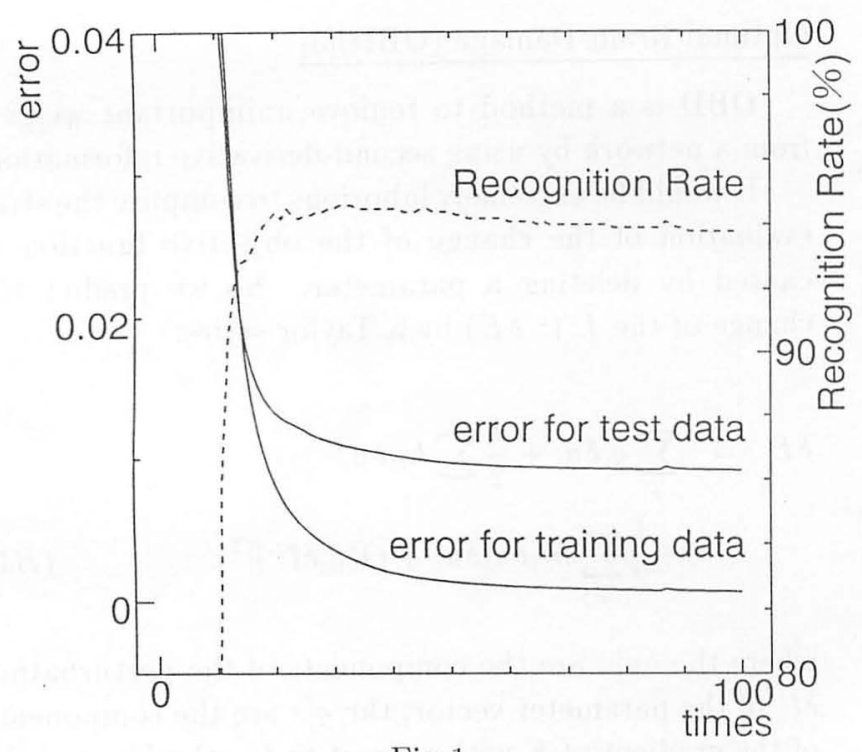

Fig.1

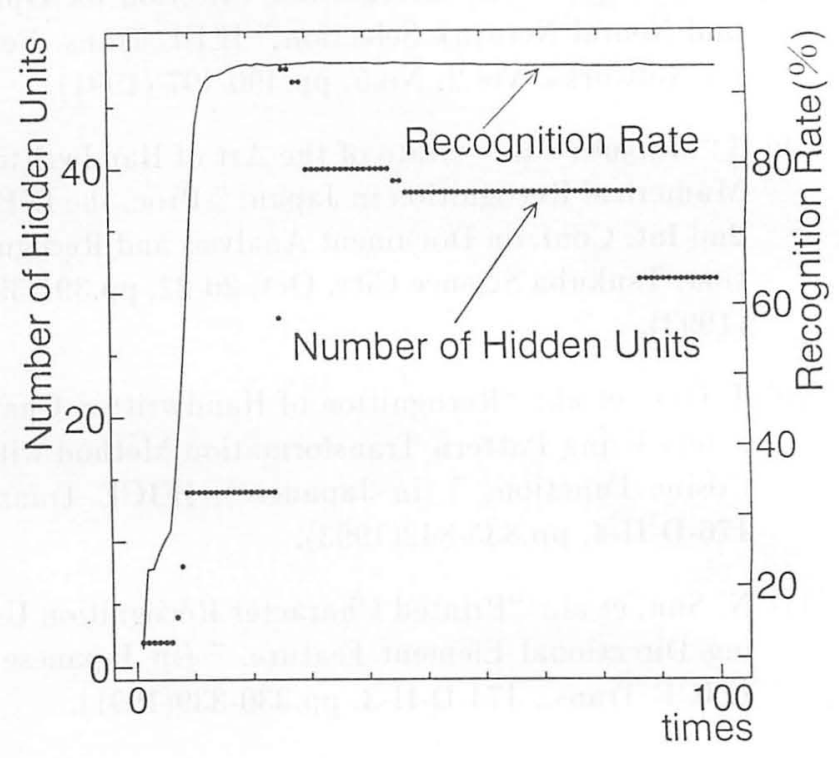

Fig.2

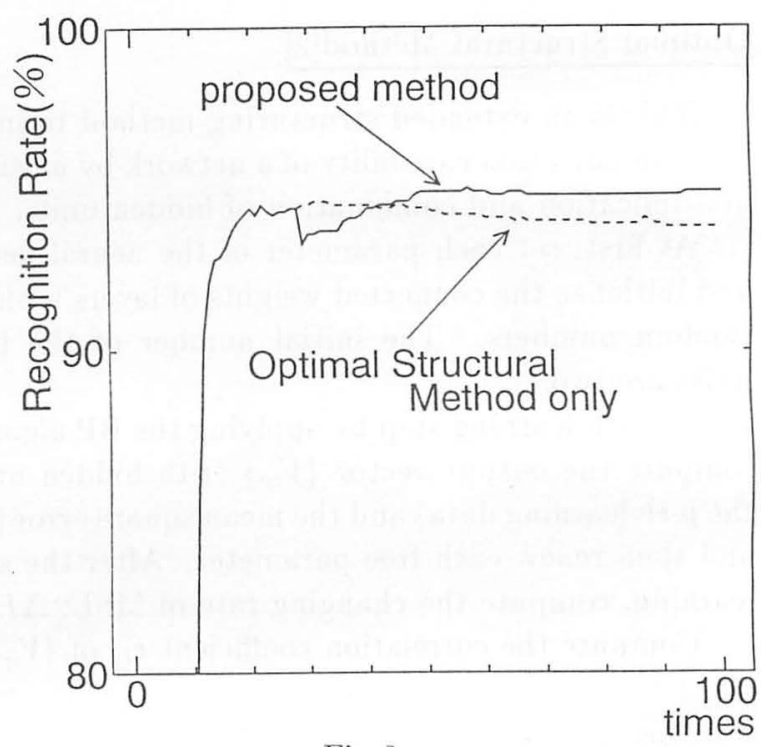

Fig.3

Table 1

\begin{tabular}{|cccrccc|}
\hline times & Units & $E$ & A I C & M D L & $E_{\text {test }}$ & $(\%)$ \\
\hline 27 & 48 & 0.004403 & -76696.0 & -27099.2 & 0.011573 & 94.4 \\
29 & 47 & 0.003824 & -78664.4 & -28317.3 & 0.011268 & 94.5 \\
44 & 40 & 0.001692 & -88068.9 & -34657.0 & 0.009981 & 94.4 \\
46 & 39 & 0.001576 & -89244.1 & -35478.5 & 0.009868 & 94.5 \\
86 & 38 & 0.000762 & -101384.7 & -41782.7 & 0.009181 & 93.9 \\
\hline
\end{tabular}

